\title{
POWERS OF THREE AS DIFFERENCE OF TWO FIBONACCI NUMBERS $\left(F_{n}-F_{m}=3^{p}\right)$
}

\section{Pagdame Tiebekabe and Ismaïla Diouf}

Université Cheikh Anta Diop (UCAD)

Laboratoire d'Algèbre, de Cryptologie, de Géométrie Algébrique

et Applications (LACGAA)

Dakar, Sénégal

\begin{abstract}
The goal of this paper is to find, in a simple and rigorous way, all powers of three as the difference of two Fibonacci numbers, that is, we study a diophantine equation $F_{n}-F_{m}=3^{p}$ in nonnegative integers $n, m$, and $p$ where $\left(F_{n}\right)_{n \geq 0}$ is the Fibonacci sequence. The tools used to solve our main result are properties of continued fractions, linear forms in logarithms, and a version of the Baker-Davenport reduction method in diophantine approximation.
\end{abstract}

\section{Introduction}

The Fibonacci and Lucas numbers 1, 1, 2, 3, 5, 8, ... and 2, 1, 3, 4, 7, 11, ... respectively have fascinated mathematicians for over hundred years. Fibonacci and Lucas sequences are defined recursively by $F_{n}=F_{n-1}+$ $F_{n-2}$ for $n \geq 2$. They are generated by $F_{0}=1, F_{1}=1$ and $F_{0}=2, F_{1}=1$

Received: January 8, 2021; Revised: January 22, 2021; Accepted: January 25, 2021

2020 Mathematics Subject Classification: Kindly provide.

Keywords and phrases: linear forms in logarithm, diophantine equations, fibonacci sequence, Lucas sequence. 
respectively and belong to a list of particular member of a general family of sequences. The determination of perfect powers of Lucas and Fibonacci sequences does not date from today. They have many interesting properties and have been studied by many researchers. For a brief history of Fibonacci and Lucas sequences one can consult [5]. Proprieties of Fibonacci and Lucas sequences, and the relationship between them, have been the focus of a considerable amount of research. The real contribution of determination of perfect powers of Lucas and Fibonacci sequences began in 2006. By classical and modular approaches of Diophantine equations, Bugeaud et al. [8] defined all perfect powers of Lucas and Fibonacci sequences by solving the equations $L_{n}=y^{p}$ and $F_{n}=y^{p}$. Firstly, square terms and later perfect powers in the Fibonacci and Lucas sequences have attracted the attention of the researchers. From there, many researchers tackled similar problems.

It is important to recall that, in Corollary 1 of [1], Kebli et al. give the condition on all integer solutions of equation $F_{n} \pm F_{m}=y^{a}$. They give also in Theorem 1 and Theorem 2 of [1], the conditions $F_{n} \pm F_{m}=y^{a}$ must be satisfied to have finitely many nonnegative integer solution $(n, m, y, a)$. The proof of their Theorem 2 is based on abc-conjecture (open problem). But it also uses an upper bound on $n-m$ which appears in the proof of Theorem 1 of [1] and rests on an application of lower bounds for linear forms in logarithms of algebraic numbers. One can consult [1] for more information. Our results, respect all conditions given in [1].

Motivated by the studies of Bravo and Luca [1, 2], our main focus is on the powers of 3 as a difference of two Fibonacci numbers. We consider the Diophantine equation

$$
F_{n}-F_{m}=3^{p}
$$

in nonnegative integers $m, n$, and $p$. We follow the approach and the method presented By Luca and Bravo in [2]. The proof of our main result uses lower bounds for linear forms in logarithms, properties of continued fractions, and 
a version of the Baker-Davenport reduction method in diophantine approximation.

This paper follows the following steps: In Section 2, we introduce necessary theorems and lemma used in Section 3 to prove the main result of this paper stated below:

Theorem 1. The only solutions $(n, m, p)$ of the Diophantine equation $F_{n}-F_{m}=3^{p}$ in nonnegative integers $m<n$ and $p$ are

$$
\begin{aligned}
& (1,0,0), \quad(2,0,0), \quad(3,1,0), \quad(3,2,0), \quad(4,0,1), \\
& (4,3,0), \quad(5,3,1), \quad(6,5,1), \quad(11,6,4) .
\end{aligned}
$$

\section{Auxiliary Results}

In this section, we recall some important results used by us. The wellknown Binet formulas provide closed form rules to calculate the Fibonacci and Lucas numbers, both incorporate the so-called golden ratio $(1+\sqrt{5}) / 2$, hinting at a deep connection between the sequences:

$$
F_{n}=\frac{\alpha^{n}-\beta^{n}}{\sqrt{5}} \text { and } L_{n}=\alpha^{n}+\beta^{n}
$$

where $\alpha=(1+\sqrt{5}) / 2$ and $\beta=(1-\sqrt{5}) / 2$ are the roots of the characteristic equation $x^{2}-x-1=0$. The relation between Fibonacci and Lucas numbers and $\alpha$ are:

$$
\begin{aligned}
& F_{n+1}+F_{n}=L_{n}, \\
& \alpha^{n-2} \leq F_{n} \leq \alpha^{n-2}, \text { for } n \geq 1 .
\end{aligned}
$$

(3) can be proved easily by induction, and together with (2) allows us, in Section 3, to prove the following important inequality: $1 \leq p<n$. It can be seen that $1<\alpha<2$ and $-1<\beta<0$.

The following theorem due to Lucas and Patel is given in [4]. 
Theorem 2. All solutions of Diophantine equation $F_{n} \pm F_{m}=y^{p}$, $p \geq 2$ in integers $(n, m, y, p)$ with $n \equiv m(\bmod 2)$ have either $\max \{|n|,|m|\}<36$ or $y=0$ and $|n|=|m|$.

Theorem 2 is a generalization of the following theorem due to Bugeaud et al. [8].

Theorem 3. The only nonnegative solutions $(n, y, p)$ of the equation $F_{n}-1=y^{p}$ with $p \geq 2$ are

$$
F_{1}-1=F_{2}-1=0, \quad F_{3}-1=1, \quad F_{5}-1=2^{2} .
$$

We also need the following theorem due to Matveev and Lemma due to Dujella and Pethö [6, 7].

Theorem 4 (Matveev [6]). Let $n \geq 1$ be an integer. Let $\mathbb{L}$ be a field of algebraic numbers of degree $D$. Let $\eta_{1}, \ldots, \eta_{l}$ be non-zero elements of $\mathbb{L}$ and let $b_{1}, b_{2}, \ldots, b_{l}$ be integers, where

$$
B:=\max \left\{\left|b_{1}\right|, \ldots,\left|b_{l}\right|\right\},
$$

and

$$
\Lambda:=\eta_{1}^{b_{1}} \cdots \eta_{l}^{b_{l}}-1=\left(\prod_{i=1}^{l} \eta_{i}^{b_{i}}\right)-1 .
$$

Let $A_{1}, \ldots, A_{l}$ reals numbers such that

$$
A_{j} \geq \max \left\{D h\left(\eta_{j}\right),\left|\log \left(\eta_{j}\right)\right|, 0.16\right\}, 1 \leq j \leq l .
$$

Assume that $\Lambda \neq 0$, then we have

$\log |\Lambda|>-3 \times 30^{l+4} \times(l+1)^{5.5} \times d^{2} \times A_{1} \cdots A_{l}(1+\log D)(1+\log n B)$.

Further, if $\mathbb{L}$ is real, then

$$
\log |\Lambda|>-1.4 \times 30^{l+3} \times(l)^{4.5} \times d^{2} \times A_{1} \cdots A_{l}(1+\log D)(1+\log B) .
$$


During our calculations, we get upper bounds on our variables which are too large, so we have to reduce them. To do this, we use some results from the theory of continued fractions. In particular, for a non-homogeneous linear form with two integer variables, we use a slight variation of a result due to Dujella and Pethö [7]. For a real number $X$, we write $\|X\|:=$ $\min \{|X-n|: n \in \mathbb{Z}\}$ for the distance of $X$ to the nearest integer.

Lemma 5 (Dujella and Pethö [7]). Let $M$ a positive integer, let $p / q$ be the convergent of the continued fraction of the irrational number $\gamma$ such that $q>6 M$ and let $A, B, \mu$ be real numbers such that $A>0$ and $B>1$. Let $\varepsilon:=\|\mu q\|-M\|\gamma q\|$. If $\varepsilon>0$, then the inequality

$$
0<m \gamma-n+\mu<A B^{-k}
$$

does not admit a solution in integers $m, n$ and $k$ such that $m \leq M$ and $k \geq \frac{\log (A q / \varepsilon)}{\log B}$.

The following properties of the logarithmic height is used in the next section:

- $h(\gamma \pm \eta) \leq h(\gamma)+h(\eta)+\log 2$

- $h\left(\gamma \eta^{ \pm 1}\right) \leq h(\gamma)+h(\eta)$

- $h\left(\gamma^{s}\right)=|s| h(\gamma)$.

\section{Main Result}

In this section, we give the proof of Theorem 1. The only solutions $(n, m, p)$ of the Diophantine equation $F_{n}-F_{m}=3^{p}$ in nonnegative integers $m<n$ and $p$ are

$$
\begin{aligned}
& (1,0,0), \quad(2,0,0), \quad(3,1,0), \quad(3,2,0), \quad(4,0,1), \\
& (4,3,0), \quad(5,3,1), \quad(6,5,1), \quad(11,6,4) .
\end{aligned}
$$


Proof. Assume that

$$
F_{n}-F_{m}=3^{p}
$$

holds.

If $m=0$, (1) becomes $F_{n}=3^{p}$, the only solutions $(n, m, p)$ are $(1,0,0),(2,0,0)$ and $(4,0,1)$, and $n \leq 12$ according to Theorem 2 in [4].

If $m=1$ or 2 , then we have the equation $F_{n}-1=3^{p}$. By Theorem 3, we have

$$
(n, m, p) \in\{(3,1,0),(3,2,0)\}
$$

Now assume that $n-m \in\{1,2\}$. Then (1) becomes $3^{p}=F_{n-2}$ for $m=n-1$ or $3^{p}=F_{n-1}$ for $m=n-2$, according to [4], $(n, m, p)=$ $(2,0,0),(3,1,0),(3,2,0),(4,3,0),(5,3,1),(6,5,1)$. With the aid of Mathematica, we obtain the solutions in the statement for $1 \leq m<n \leq 200$.

For now, assume $n>200, m \geq 3$ and $n-m \geq 3$. Let us prove an inequality which is important for our purpose. From $\alpha^{n-2} \leq F_{n} \leq \alpha^{n-1}$, we get

$$
3^{p}=F_{n}-F_{m}<F_{n}<\alpha^{n}<3^{n} \text { i.e., } 3^{p}<3^{n} .
$$

Since $m>3$ and $n-m \geq 3$, it follows that

$$
3^{p}=F_{n}-F_{m} \geq F_{m+3}-F_{m}=2 F_{m+1} \geq 6
$$

using (2). From (5) and (6), we get $1 \leq p<n$.

Now let assume that $n \equiv m(\bmod 2)$. This case is completely solved by Luca and Patel [4], and they get $n \leq 36$. This contradicts our assumption $n>200$. Hence (1) has no solution when $n$ and $m$ have same parities.

We now assume that $n$ and $m$ have different parities. 
$F_{n}-F_{m}=3^{p} \Rightarrow\left|\frac{\alpha^{n}}{\sqrt{5}}-3^{p}\right|=\left|F_{m}+\frac{\beta^{n}}{\sqrt{5}}\right|<\alpha^{m}+\frac{\beta^{n}}{\sqrt{5}}<\alpha^{m}+\frac{1}{2}$.

Dividing both sides, by $\frac{\alpha^{n}}{\sqrt{5}}$, we get

$$
\left|1-3^{p} \sqrt{5} \alpha^{-n}\right|<4 / \alpha^{n-m} .
$$

Now applying Matveev's theorem with the following data:

$$
\gamma_{1}:=3, \gamma_{2}:=\alpha, \gamma_{3}:=\sqrt{5}, b_{1}:=p, b_{2}:=-n \text {, and } b_{3}:=1 \text {. }
$$

Since $\gamma_{1}, \gamma_{2}, \gamma_{3} \in \mathbb{Q}(\sqrt{5})$. Then we can take $\mathbb{K}=\mathbb{Q}(\sqrt{5})$. So, $D=\operatorname{deg}\left(x^{2}-5\right)=2 . \Lambda_{1}=3^{p} \sqrt{5} \alpha^{-n}-1$ is nonzero. Otherwise, we get

$$
3^{p}=\frac{\alpha^{p}}{\sqrt{5}}=F_{n}+\frac{|\beta|^{n}}{\sqrt{5}}>F_{n}-1>F_{n}-F_{m}=3^{p} \text { which is impossible. }
$$

or we can prove it is nonzero by using the conjugate of the relation $3^{p}=\frac{\alpha^{p}}{\sqrt{5}}$.

Moreover $h\left(\gamma_{1}\right)=\log 3, h\left(\gamma_{2}\right)=\frac{1}{2} \log \alpha, h\left(\gamma_{3}\right)=\log \sqrt{5}$. Then we can take, $A_{1}=2.2, A_{2}=0.5$, and $A_{3}=1.7$. Since $p<n$, it follows that $B:=\max \{|p|,|-n|, 1\}=n$. Since all conditions are satisfied, then from Theorem 4, we have

$$
\begin{aligned}
\frac{4}{\alpha^{n-m}} & >\left|\Lambda_{1}\right| \\
& >\exp \left(-1.4 \times 30^{6} \times 3^{4.5} \times 2^{2}(1+\log 2)(1+\log n) \times 2.2 \times 0.5 \times 1.7\right)
\end{aligned}
$$

hence

$$
\begin{aligned}
& (n-m) \log \alpha-\log 4 \\
< & 1.4 \times 30^{6} \times 3^{4.5} \times 2^{2}(1+\log 2)(1+\log n) \times 2.2 \times 0.5 \times 1.7
\end{aligned}
$$


and a quick computation in Mathematica yields for

$$
(n-m) \log \alpha<2.16 \times 10^{12}(1+\log n)<3.6 \times 10^{16} \log n,
$$

where we used inequality $1+\log n<2 \log n$ which holds for all $n \geq 3$. In order to find the bound of $n$, let us rewrite equation (1) and apply Matveev's theorem second time.

$$
F_{n}-F_{m}=3^{p} \Rightarrow \frac{\alpha^{n}\left(1-\alpha^{m-n}\right)}{\sqrt{5}}-3^{p}=\frac{\beta^{n}-\beta^{m}}{\sqrt{5}} .
$$

Taking absolute value on the above equation and using the fact that $|\beta|^{n}+|\beta|^{m}<\frac{2}{3} ; n>200$; we get

$$
\left|\frac{\alpha^{n}\left(1-\alpha^{m-n}\right)}{\sqrt{5}}-3^{p}\right| \leq \frac{|\beta|^{n}+|\beta|^{m}}{\sqrt{5}}<\frac{1}{3}
$$

hence

$$
\left|1-3^{p} \sqrt{5}\left(1-\alpha^{m-n}\right)^{-1} \alpha^{-n}\right|<3 / \alpha^{n}
$$

because

$$
\alpha^{m-n}=\frac{1}{\alpha^{n-m}}<\frac{1}{\alpha}<\frac{2}{3} \Rightarrow 1-\alpha^{m-n}>\frac{1}{3} \Rightarrow\left(1-\alpha^{m-n}\right)^{-1}<3 .
$$

From (9), taking $\gamma_{1}:=3, \gamma_{2}:=\alpha, \gamma_{3}:=\sqrt{5}\left(1-\alpha^{m-n}\right)^{-1} ; b_{1}:=p, b_{2}:=$ $-n, b_{3}:=1$. Since $\gamma_{1}, \gamma_{2}, \gamma_{3} \in \mathbb{Q}(\sqrt{5})$. Then we can take $\mathbb{K}=\mathbb{Q}(\sqrt{5})$. So, $D=\operatorname{deg}\left(x^{2}-5\right)=2 . \quad \Lambda_{2}:=3^{p} \sqrt{5}\left(1-\alpha^{m-n}\right)^{-1} \alpha^{-n}-1$. Let prove that $\Lambda_{2} \neq 0$ by contradiction. If $\Lambda_{2}=0$, then we have

$$
3^{p}=\frac{\alpha^{n}-\alpha^{m}}{\sqrt{5}} \Rightarrow \frac{\beta^{n}}{\sqrt{5}}=\frac{\beta^{m}}{\sqrt{5}} \text { which is impossible since } n>m \text {. }
$$

Similarly

$$
h\left(\gamma_{1}\right)=\log 3, h\left(\gamma_{2}\right)=\frac{1}{2} \log \alpha, h\left(\gamma_{3}\right) \leq \log 2 \sqrt{5}+\frac{1}{2}(n-m) \log \alpha
$$


and so we take $A_{1}=2.2, A_{2}=0.5, A_{3}=\log 20+(n-m) \log \alpha$. Also, since $p<n$, then $B:=\{p,|-n|, 1\}=n$. This implies

$$
\begin{aligned}
\frac{3}{\alpha^{n}} & >\left|\Lambda_{2}\right| \\
& >\exp ((-C)(1+\log 2)(1+\log n) \cdot 2.2 \cdot 0.5 \cdot(\log 20+(n-m) \log \alpha))
\end{aligned}
$$

or

$$
\begin{aligned}
& n \log \alpha-\log 3 \\
< & C(1+\log 2)(1+\log n) \cdot 2.2 \cdot 0.5 \cdot(\log 20+(n-m) \log \alpha),
\end{aligned}
$$

where $C=1.4 \cdot 30^{6} \cdot 3^{4.5} \cdot 2^{2}$. Replacing (8) in (10), we get

$$
\begin{aligned}
& n \log \alpha-\log 3 \\
< & C(1+\log 2)(1+\log n) \times 2.2 \times 0.5 \times\left(\log 20+3.6 \times 10^{16} \log n\right)
\end{aligned}
$$

and so $n<4.6 \times 10^{32}$. Our objective now, is to reduce the upper bound on $n$ obtained. For this, let us apply Lemma of Dujella and Pethö. From (7), let

$$
z_{1}:=p \log 3-n \log \alpha+\log \sqrt{5} .
$$

Then $\left|1-e^{Z_{1}}\right|<4 / \alpha^{n-m}$ by (8). The inequality

$$
\frac{\alpha^{n}}{\sqrt{5}}=F_{n}+\frac{\beta^{n}}{\sqrt{5}}>F_{n}-1>F_{n}-F_{m}=3^{p}
$$

implies that $z_{1}<0$. In that case, since $4 / \alpha^{n-m}<0.95$ for $n-m \geq 3$, it follows that $e^{\left|z_{1}\right|}<20$, hence we get

$$
0<\left|z_{1}\right|<e^{\left|z_{1}\right|}-1 \leq e^{\left|z_{1}\right|}\left|1-e^{z_{1}}\right|<80 / \alpha^{n-m}
$$

or

$$
0<|p \log 3-n \log \alpha+\log \sqrt{5}|<80 / \alpha^{n-m}
$$


then, by dividing the above equation by $\log \alpha$, we get

$$
0<\left|p\left(\frac{\log 3}{\log \alpha}\right)-n+\frac{\log \sqrt{5}}{\log \alpha}\right|<167 \cdot \alpha^{-(n-m)}
$$

Now we can apply lemma with the following data: It is clear that $\gamma:=\frac{\log 3}{\log \alpha}$ is irrational.

$$
\text { Let } \mu:=\frac{\log \sqrt{5}}{\log \alpha}, A:=167, B:=\alpha, k:=n-m \text {. Taking } M:=4.6 \times 10^{32} \text {, }
$$
we find after computation in SageMath,

$$
q_{65}=14312730362266447314022834209225543>6 M
$$

and

$$
n-m \leq \frac{\log \left(A q_{65} / \varepsilon\right)}{\log B}<84.7638
$$

by Lemma 5. Moreover $n-m<85$.

Substituting this upper bound for $n-m$ into (10), we get $n<3.58$ $\times 10^{15}$.

Now, from (9), let $z_{2}=p \log 3-n \log \alpha+\log \left(\sqrt{5}\left(1-\alpha^{m-n}\right)^{-1}\right)$. In this case,

$$
\left|1-e^{z_{2}}\right|<3 / \alpha^{n}
$$

It seems that $3 / \alpha^{n}<1 / 2$. Two cases arise: when $z_{2}>0$ and $z_{2}<0$.

- Case 1. $z_{2}>0$.

Then $0<z_{2}<e^{z_{2}}-1<3 / \alpha^{n}$.

- Case 2. $z_{2}<0$. 
Then $\left|1-e^{z_{2}}\right|=1-e^{z_{2}}<3 / \alpha^{n}<1 / 2 \Rightarrow e^{z_{2}}<2$ and therefore

$$
0<\left|z_{2}\right|<e^{z_{2}}-1=e^{\left|z_{2}\right|}\left|1-e^{z_{2}}\right|<6 / \alpha^{n} \Rightarrow 0<\left|z_{2}\right|<6 / \alpha^{n}
$$

this is equivalent to

$$
0<\left|p \log 3-n \log \alpha+\log \left(\sqrt{5}\left(1-\alpha^{m-n}\right)^{-1}\right)\right|<6 / \alpha^{n}
$$

thus

$$
0<\left|p \frac{\log 3}{\log \alpha}-n+\frac{\log \left(\sqrt{5}\left(1-\alpha^{m-n}\right)^{-1}\right)}{\log \alpha}\right|<13 \cdot \alpha^{n}
$$

Putting $\gamma=\frac{\log 3}{\log \alpha} ;$ and taking $M=3.58 \times 10^{15}$; we find with $\mu=\frac{\log \left(\sqrt{5}\left(1-\alpha^{m-n}\right)^{-1}\right)}{\log \alpha}$, for $n-m \in[3,84], A=13, B=\alpha, k=n$ by Lemma 5, that $q_{40}=4488642667247434242$ and so $n \leq \frac{\log \left(A q_{40} / \varepsilon\right)}{\log B} \leq 80$.

This contradicts our assumption $n>200$. This ends the proof of our main theorem.

\section{References}

[1] S. Kebli, O. Kihel, J. Larone and F. Luca, On the nonnegative integer solutions to the equation $F_{n} \pm F_{m}=y^{a}$, J. Number Theory 220 (2021), 107-127.

[2] J. J. Bravo and F. Luca, On the Diophantine equation $F_{n}+F_{m}=2^{a}$, Quaest. Math. 39 (2016), 391-400.

[3] Y. Bugeaud, M. Mignotte and S. Siksek, Classical and modular approaches to exponential Diophantine equations I. Fibonacci and Lucas perfect powers, Ann. of Math. 163 (2006), 969-1018.

[4] F. Luca and V. Patel, On perfect powers that are sums of two Fibonacci numbers, J. Number Theory 189 (2018), 90-96. 
[5] L. Debnath, A short history of the Fibonacci and golden numbers with their applications, Int. J. Math. Education Sci. Technology 42 (2011), 337-367.

[6] E. M. Matveev, An explicit lower bound for a homogeneous rational linear form in the logarithms of algebraic numbers, II, Izv. Ross. Akad. Nauk Ser. Mat. 64 (2000), 125-180. Translation in Izv. Math. 64 (2000), 1217-1269.

[7] A. Dujella and A. Pethö, A generalization of a theorem of Baker and Davenport, Quart. J. Math. Oxford Ser. 49(195) (1998), 291-306.

[8] Y. Bugeaud, F. Luca, M. Mignotte and S. Siksek, Fibonacci numbers at most one away from a perfect power, Elem. Math. 63 (2008), 65-75. 
Paper No. PPH-2101018-ANT

Kindly return the proof after correction to:

The Publication Manager

Pushpa Publishing House

Vijaya Niwas

198, Mumfordganj

Prayagraj-211002 (India)

along with the print charges*.

*Invoice attached
Proof read by:

Copyright transferred to the Pushpa Publishing House

Signature:

Date:

Tel:

e-mail:

Number of additional reprints required

Cost of a set of 25 copies of additional reprints@ U.S. Dollars 15.00 per page.

(25 copies of reprints are provided to the corresponding author ex-gratis) 